\title{
La sequenza sismica dell'Italia centrale del 24 agosto e successive: contributi alla conoscenza e la banca dati degli effetti di superficie
}

\author{
Piero Farabollini (a), Serafino Angelini (b), Massimiliano Fazzini (a), Francesca Romana Lugeri (c), \\ Gianni Scalella (d) \& GeomorphoLab (a*)
}

\begin{abstract}
(a) Scuola di Scienze e Tecnologie, Sezione di Geologia, Università degli Studi di Camerino, Via Gentile da Varano, 1, 62032 Camerino (MC).
(b) CIA Lab S.r.l., Via Mutilati e Invalidi del Lavoro, 29, 63100 Ascoli Piceno.

(c) Servizio Geologico d'Italia - ISPRA, Via Vitaliano Brancati, 48, 00144 Roma.

(d) Regione Marche, via Gentile da Fabriano, 9, 60125 Ancona.

(*) Al Gruppo GeomorphoLab afferiscono i seguenti ricercatori (in ordine alfabetico): D. Aringoli (Unicam), S. Angelini (libero professionista), M. Bufalini (Unicam), P. Farabollini (Unicam), M. Fazzini (Unicam), E. Fuffa (Unicam), M. Giacopetti (Unicam), F. R. Lugeri (Ispra), M. Materazzi (Unicam), G. Pambianchi (Unicam), G. Scalella (Regione Marche).

Corresponding author e-mail: piero.farabollini@unicam.it
\end{abstract}

Document type: Short note.

Manuscript submitted 16 October 2017; accepted 16 February 2018; editorial responsibility and handling by S. Crema.

\section{ABSTRACT}

The effect of earthquakes on structures and landscape can basically be divided into two types: direct and secondary effects. The direct effects are related to the deformation of the ground at the fault whose breakage has generated the seismic event and the effects are limited to an area that is short and close to breakage.

The secondary effects resulting from the temporary passage of seismic waves may, however, also affect very large areas, causing a widespread presence of permanent effects on the Earth's surface which may affect very large areas, which, in relation to the length of the fault and the magnitude released by the event can be in the order of thousands of $\mathrm{km}^{2}$. Following the seismic events commenced on 24 August 2016 (M6.0) and continued with the shocks of October 26 (M5.4 and M5.9) until October 30 (M6.5), the Unicam GeomorphoLAB Group has mapped and computerized about 4000 permanent deformations on the Earth's surface recognized in an area of over $6000 \mathrm{~km}^{2}$ : fractures to the ground, activation and reactivation of large landslides and DGPV, dolines, mud volcanoes and liquefaction phenomena, landslides and small dams, changes in the water regime of the springs and the flow of rivers, cracks and $/$ or fractures and / or deformations on network infrastructures, etc. The present work, therefore, based on the database and the GIS on surface effects, aims at representing a cognitive and interpretative contribution to the surface evidence produced during the seismic crisis of central Italy, looking, based on correlations with the seismic events that affected Umbria-Marche in 1997 and L'Aquila in 2009, to verify and implement, if necessary, the ESI scale which, in the specific case, seems to underestimate its effects.

The analysis allowed to verify a high number of surface effects related to fracturing and seismic cracking and deformations on road and network infrastructures and, instead, a low number of activations and / or reactivations of landslides or subsidence. Although small, very significant is the number of variations of the water regime of the springs.

KEY WORDS: 2016 Central Apennine Earthquake; Database and GIS, Geomorphological deformation and coseismic effects, Central Italy.

\section{INTRODUZIONE}

A partire dal 24 agosto 2016 è iniziata nell'area del centro-Italia, tra le regioni Marche, Abruzzo, Lazio ed Umbria, una importante sequenza sismica che ha interessato un'area grande circa $5000 \mathrm{~km}^{2}$ (Fig. 1). Alla prima scossa di magnitudo 6.0, con epicentro situato nei pressi dell'abitato di Accumoli (Rieti, Lazio), ha fatto seguito circa 57.000 repliche con altri quattro importanti eventi sismici, rispettivamente pari a $5.7 \mathrm{M}, 5.9 \mathrm{M}, 6.5 \mathrm{M}$ e $5.7 \mathrm{M}$ (rispettivamente il 26 ottobre, il 30 ottobre 2016 alle ore 19,10 e 21,30 circa; il 18 gennaio 2017).

A seguito dell'evento principale, sono state subito avviate verifiche sugli effetti ambientali del terremoto legati alla riattivazione del sistema di faglie, ipotizzate come le "faglie del terremoto", nonché sugli effetti secondari legati alla scossa principale (ad es. frane, fratture al suolo, variazioni delle caratteristiche idrogeologiche delle sorgenti, sinkhole, ecc.) alfine di mappare e catalogare tutte le evidenze. Alcuni di questi studi sono stati già oggetto di recenti pubblicazioni o report scientifici (Farabollini et al., 2016; Aringoli et al., 2016; Farabollini et al., 2017; Civico et al., 2018); nel presente lavoro invece vengono riportati i risultati della realizzazione della bancadati, ed il GIS ad esso associato, realizzata appositamente per l'enorme quantità di dati acquisiti.

\section{CARATTERISTICHE GEOLOGICHE E SISMICHE DELL'AREA}

Il bacino del Castelluccio è una depressione intramontana, situata nell'Appennino centrale e riempita da sedimenti fluvio-lacustri deposti durante il Pleistocene medio fino all'Olocene (Coltorti \& Farabollini, 2002); le unità di base sono invece rappresentate da calcari micritici e pelagici di età Giurassico-Miocene (Pierantoni et al., 2012; Fig. 1).

La principale modellazione geomorfologica del paesaggio è iniziata durante il Pliocene finale, quando condizioni climatiche umide o subtropicali, favorevoli ai processi di pianificazione, hanno creato un paleopaesaggio a bassa energia di rilievo.

La fase tettonica successiva, attiva dal Pleistocene inferiore-medio fino ad ora, è caratterizzata da faglie 


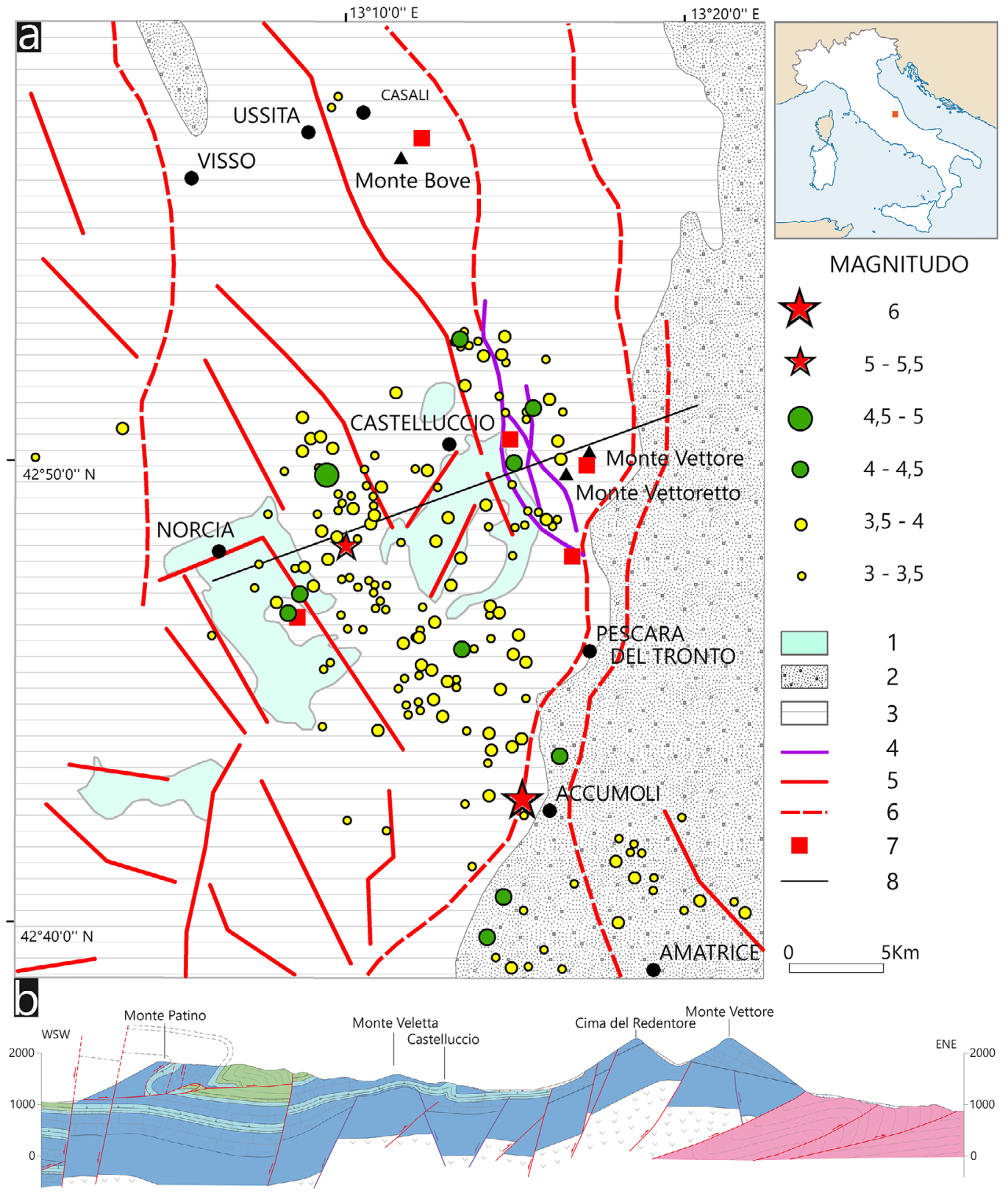

Fig.1 - a) Schizzo geologico e strutturale dell'area di studio con ubicazione dei principali epicentri: 1) Depositi continentali quaternari; 2) Bedrock prevalentemente arenaceo-pelitico; 3) Bedrock prevalentemente calcareo e calcareo marnoso; 4) Sistema di faglie del Monte Vettore; 5) Faglie dirette appenniniche ed antiappenniniche; 6) Thrusts: 7) principali DSGSD e grandi frane; 8) tracce di sezione (vedi sotto). b) Sezione schematica: 1) formazioni prevalentemente calcaree; 2) formazioni calcareomarnose; 3) formazioni marnose e calcareo-marnose; 4) formazioni arenaceo-pelitiche. Carta geologica schematica e profilo geologico (modificata da Aringoli et al., 2016). dip-slip ed oblique e da forte entità del sollevamento. Questi processi hanno interrotto e disarticolato il precedente paesaggio, formando una serie di depressioni tettoniche, quali Colfiorito, Castelluccio, Norcia, Cascia, Leonessa, ecc., che caratterizzano tutta l'area appenninica (Coltorti \& Farabollini, 2002 cum bib.; Aringoli et al., 2016). Il sollevamento quaternario prodottosi, insieme all'assetto stratigrafico, ha generato una intensa attività morfodinamica che ha portato alla rimodellazione significativa del paesaggio precedente, attivando anche grandi frane e profonde deformazioni gravitative profonde di versante o DSGSD (Aringoli et al., 1996; Aringoli et al., 2010b), alcune delle quali hanno mostrato prove di riattivazione durante l'attuale sequenza sismica.

Per quanto riguarda la sismicità, sono stati documentati due principali terremoti distruttivi storici e strumentali, il 14 gennaio 1703 (X MCS, Mw =6.6) e il 19 settembre $1979($ Ms = 5.9, profondità focale di $6-8 \mathrm{~km})$ anche se molti altri si sono verificati nel tempo, soprattutto nel secolo diciottesimo (Rovida et al., 2016), ma con magnitudo molto minori, dati dal basso grado di danneggiamento segnalato. I dati di errore e i meccanismi focali del terremoto mostrano un'estensione NE-SW preesistente, ma sono stati anche determinati meccanismi strike-slip e inversi (Cello et al., 1997). Questi terremoti storici, compreso quello recente, sono stati associati all'attività di un sistema di faglie attive che corre in direzione NNW-SSE da Colfiorito all'Aquila ed interpretato come l'espressione superficiale di faglie sismogenetiche profonde (Tondi \& Cello, 2003).

Il versante del Monte Vettore è caratterizzato dalla presenza di due faglie normali: la faglia più bassa è presente alla base della Scarpata del Vettore e limita il bacino del Castelluccio. La faglia più in alto invece, corre molto vicino alla cima del Mt. Redentore, ed è contrassegnata da una scarpata di faglia chiaramente visibile per una lunghezza di circa $7 \mathrm{~km}$, comunemente chiamata "Cordone del Vettore" 
e riportata anche nella cartografia IGM del 1957. Infatti il versante occidentale del Monte Vettore è l'espressione morfologica di tale elemento tettonico di immersione SWNNW-SSE (lunghezza totale $30 \mathrm{~km}$ ) con terminazione settentrionale a Mt. Bove (Pierantoni et al., 2013).

\section{EFFETTI GEOLOGICI COSISMICI}

Subito dopo gli eventi del 24 agosto e, successivamente, dopo quelli di ottobre e di gennaio, il Gruppo Geomorpholab dell'Università di Camerino, con colleghi geologi della Regione Marche e di altri Enti di Ricerca (DPCN, INGV e ISPRA), si è mosso sul territorio alfine di fotografare, cartografare, classificare, catalogare ed informatizzare, attraverso piattaforma GIS, le innumerevoli evidenze di superficie legate allo scuotimento sismico nell'area del cratere. Gli effetti diretti sono collegati alla deformazione del suolo in corrispondenza della faglia la cui rottura ha generato l'evento sismico e gli effetti sono limitati ad un'area poco estesa e prossima alla rottura (Civico et al., 2018).

Gli effetti secondari, risultanti dal passaggio temporaneo delle onde sismiche, sono stati riconosciuti anche molto lontano dagli epicentri, causando una diffusa presenza di evidenze permanenti sulla superficie terrestre che, in rapporto alla lunghezza della faglia ed alla magnitudo liberata dall'evento, sono state valutate dell'ordine delle migliaia di $\mathrm{km}^{2}$. Sono state raccolte più di 4000 evidenze che hanno permesso di costruire una banca-dati sugli effetti del terremoto distinti in effetti diretti ed effetti secondari (Farabollini et al., 2017): fratture al suolo, attivazione e riattivazione di grandi frane e DGPV, collassi di doline; vulcanelli di fango e fenomeni di liquefazione, sbarramenti per frana, cedimenti differenziali, modificazioni del regime idrico delle sorgenti e della portata dei fiumi, fessure e/o fratture e/o deformazioni su infrastrutture viarie ed a rete, ecc. (Fig. 2).

Le osservazioni sugli effetti geomorfologici provocati dal sisma evidenziano una stretta correlazione e casistica con quelli che si sono prodotti in occasione di precedenti eventi sismici che hanno interessato l'appennino centrale, quali ad es. la sequenza sismica Umbria-Marche, 1997 o quella de L'Aquila, 2009, anche se quest'ultimi hanno mostrato evidenze molto minori e diffuse in un territorio notevolmente più limitato.

Diseguitosiriportanole principali evidenzericonosciute nell'area del "cratere" cercando, quando possibile ed in base anche alla loro importanza morfogenetica e morfodinamica, di descriverne le caratteristiche peculiari che le contraddistinguono e permettono di classificarle come effetti cosismici.

1 - Riattivazione di grandifrane e deformazioni gravitative profonde di versante. In un precedente studio (Farabollini et al., 1995) era stato evidenziato che nell'area appenninica erano state riconosciute circa 500 grandi frane e/o DGPV delle quali 200 rientrano nell'area del cosiddetto cratere della sequenza sismica del 24 agosto e successive. L'analisi condotta su queste ultime ha evidenziato che circa il $25 \%$ di esse ha subito una riattivazione, sia in termini di fenomeno generale che in termini di singoli elementi morfologici.

Nell'area di Passo Cattivo, alla sommità della valle del Tenna, sono stati riconosciuti elementi tipici delle deformazioni gravitative profonde di versante che hanno avuto anche risentimenti superficiali, sempre legati alla gravità. In particolare, in cresta al versante Cima Vallinfante - Monte Porche, sono riconoscibili una serie di trincee dalla forma arcuata, parallele tra loro e via via di minore dimensione ed estensione procedendo verso il fondovalle del fiume Tenna, la più importante delle quali taglia la sommità del crinale stesso, già sia nelle immagini da satellite del 4 agosto 2013 e del 18 maggio 2016 e che sembrano non aver subito riattivazioni a seguito dell'evento sismico del 24 agosto 2016 ma che invece presentano una larghezza di circa 2 metri ed una profondità valutabile in circa $1,5 \mathrm{~m}$, dopo gli eventi sismici dell'ottobre 2016 (Fig. 2a).

Dopo gli eventi sismici di ottobre si sono riattivati gli estesi fenomeni franosi, soprattutto crolli e valanghe di roccia, presenti nella porzione medio-sommitale del versante di Vallinfante, creando estese conoidi detritiche alla base del versante stesso e producendo un evidente arretramento della scarpata principale, con fratture di tensione allineate secondo la forma arcuata della scarpata di frana stessa, con distacchi, pinnacoli rocciosi e blocchi aggettanti (particolare della Fig. 3); tali materiali, inoltre, potrebbero rimobilizzarsi anche a seguito dell'azione crioclastica data sia la forte fratturazione che pervade l'ammasso roccioso sia per la natura calcarea dell'ammasso stesso e delle sue caratteristiche giaciturali, leggermente a franapoggio.

Gli elementi morfologici sopra menzionati permettono di ipotizzare la presenza di una deformazione gravitazionale profonda (DSGSD) che interessa la parte più alta del versante con una profondità di alcune centinaia di metri e parzialmente coperta da materiale detritico derivante dalle frane di crollo e da processi tipo debris-flow che continuamente si verificano in tutta l'area (Farabollini \& Spurio, 2008). La genesi di questo fenomeno, che può essere definita come una deep rock creep, può essere associata all'alta energia di rilievo generata dal sollevamento tettonico quaternario, dalla importante e pervasiva interazione tra il sistema di faglia del Monte Vettore e del sovrascorrimento dei Monti Sibillini (Aringoli et al., 2016) e dalla forte fratturazione dell'ammasso roccioso.

2- Crolli di roccia elo debris avalanches: presenti soprattutto lungo versanti a luoghi anche non molto ripidi, interessando aree antropizzate e non (Montegallo, Monte Vettore, Valnerina, Sasso Spaccato). In alcuni casi questi fenomeni hanno anche prodotto lo sbarramento di fiumi con la creazione di bacini lacustri poco profondi e temporanei (Valnerina; Gola dell'Infernaccio);

3 - valanghe: sul versante sud-est del Monte Sibilla Monte Porche e Monte Bove connesse con le precipitazioni di tipo nevoso del dicembre e soprattutto del 6 gennaio e successivamente del 16-19 gennaio 2017 alle quali hanno fatto seguito scosse sismiche importanti (18 gennaio 2017); a tale propostito sono state riportate sui modelli 1 del Corpo Forestale dello Stato - Servizio Meteomont - evidenze di attività valanghive con ogni probabilità cosismica alle scosse delle 10.33 LT in località Montemonaco e Bolognola - Pintura;

4 - attivazione elo riattivazione di frane superficiali insistenti su infrastrutture viarie e a rete dell'area del "cratere" (Fig. 2b) di dimensioni a luoghi anche ragguardevoli;

5 - cedimenti elo collassi, formatisi sul Pian Grande di Castelluccio per la riattivazione di una delle numerose doline presenti nella piana, con uno sprofondamento di circa 4 metri e per una larghezza di circa 5 metri; 

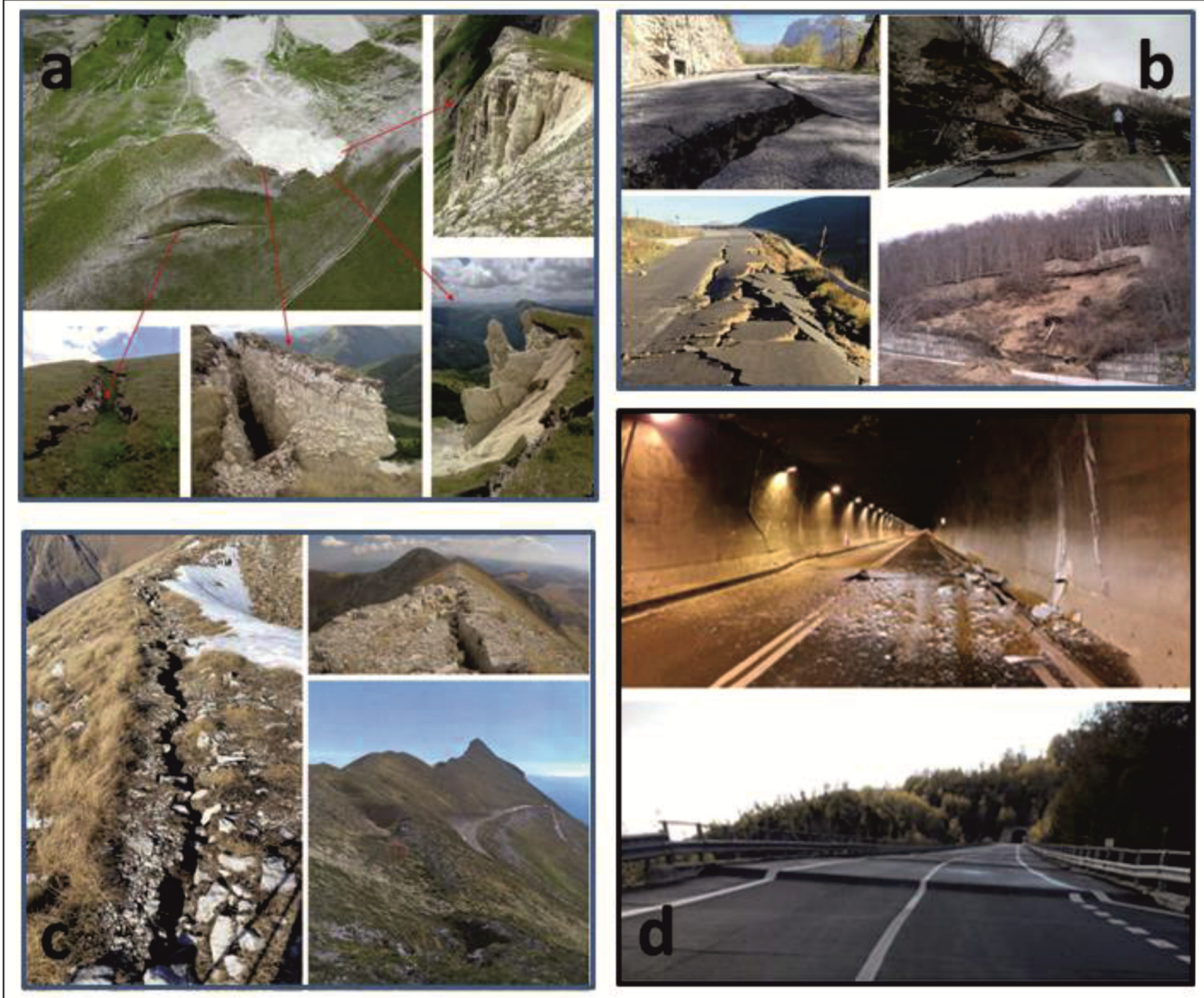

Fig. 2 - a: Immagine dall'alto dell'area di Passo Cattivo tra Monte Bicco e Monte Porche (in primo piano l'area vista dall'alto): in basso a sinistra la frattura della larghezza di circa $2 \mathrm{~m}$ e della profondità anche di 1,5m, che taglia, con una geometria arcuata, la cresta del rilievo; al centro in basso le fratture che hanno disarticolato l'ammasso roccioso creando cunei dell'ordine di quale centinaia di $\mathrm{m}^{3}$; in basso a destra speroni rocciosi aggettanti e dell'altezza di oltre.; in alto a destra la geometria della scarpata di frana, fortemente disarticolata e fratturata. La frana è stata stimata in circa $150.000 \mathrm{~m}^{3}$ di roccia. Da notare le fratture di tensione a monte della scarpata principale che evidenziano situazioni di forte instabilità del versante. b: Riattivazione di frane (in senso orario dall'alto a sinistra: Casali di Ussita; Spina di Gualdo; Castelluccio, Gualdo di Castelsantangelo sul Nera); c: A sinistra: Monte Porche; in alto a destra: Monte Argentella; sotto a destra: Monte Sibilla. Pur con soluzioni di continuità, come nel caso della Sibilla, le fratture cosismiche risultano interessare la cresta dei rilievi dei Monti Sibillini per una lunghezza anche di alcuni chilometri. d: Deformazioni del manto stradale e delle infrastrutture viarie. In basso: viadotto Capodacqua; in alto: galleria Capodacqua dove la frattura corrisponde alla dislocazione prodotta dalla faglia del Monte Vettore.

6 - fenomeni di liquefazione e vulcanelli di fango riconosciuti rispettivamente nei pressi della zona industriale a Caldarola ed in Località Curetta di S.Vittoria in Matenano;

7 - fratturazione elo fessurazione al suolo (Fig. 2c) che hanno interessato diffusamente tutta l'area epicentrale: per le prime, sicuramente le più estese, da segnalare quelle che percorrono le creste dell'allineamento Monte PorcheCima Vallelunga-Monte Argentella, a Monte Sibilla o a Monte Fema, per una lunghezza stimata di oltre $3 \mathrm{~km}$; per le seconde invece, la valutazione degli effetti deformativi permettono di stimare in quasi $1200 \mathrm{i} \mathrm{km}$ di infrastrutture viarie coinvolte da tali effetti;
8 - deformazioni al suolo (Fig. 2d) legate principalmente ad assestamenti e/o compattazione dei sedimenti quaternari ai quali sono da imputare l'elevato grado di danneggiamento di infrastrutture e di centri abitati (Pescara del Tronto, Piedilama, Pretare, Amatrice, ecc) e le numerose deformazioni delle infrastrutture viarie e/o a rete.

Da menzionare la situazione della frazione Pescara del Tronto (Fig. 3a): l'analisi geomorfologica, sia in campagna che in foto aerea, e l'analisi di dettaglio sia del substrato prequaternario che dei sedimenti quaternari di riempimento della valle del Tronto e delle loro caratteristiche geometriche e stratigrafiche, hanno permesso di evidenziare un quadro 
molto più complesso rispetto a quello che ci si aspettava sulla base della semplice osservazione di superficie o derivante da analisi della sola risposta sismica locale dei terreni. Per effetto delle forti eterogeneità delle coperture quaternarie, dato da spessori notevoli di sedimenti continentali, diversamente organizzati e cementati, con forti variazioni laterali e verticali di facies, che passano dai depositi detritici stratificati di versante, a depositi caotici di estesi corpi di frana fino a depositi travertinosi di facies "di cascata" interdigitati a depositi in facies "di cascata e vasca", è stato possibile motivare il totale crollo dell'abitato di Pescara del Tronto, rispetto al forte danneggiamento che invece ha subito l'abitato di Capodacqua, posto a pochi $\mathrm{km}$ dal primo.

L'ampia area di distribuzione degli effetti cosismici e la loro diffusa variabilità e frequenza, ha infatti permesso di comprendere come le cause della forte eterogeneità degli effetti sull'ambiente fisico e della forte diversità, a parità di caratteristiche strutturali dell'edificato, del danneggiamento, è da imputare ad un contesto geomorfologico molto articolato, dato anche da morfologie sepolte che a volte sfuggono alla sola analisi di campagna e risolvibili attraverso acquisizione di dati derivanti da indagini geostostiche specifiche.

Per quanto riguarda infine la presenza di fratture dall'andamento anche arcuato, depressioni circolari e morfologie circoidi, in aree a bassa o nulla energia di rilievo, che a luoghi ha anche condizionato l'antropizzazione del territorio, sia nelle infrastrutture che nelle attività agricole, è stata messa in relazione con la presenza di doline e/o sinkhole, sia su depositi quaternari (Pian Grande di Castelluccio) che su substrato calcareo (Monte BoveMonte Porche-Monte Sibilla).

Tali morfologie, legate a processi carsici che hanno interessato il substrato calcareo dell'area coinvolta dal sisma, molto spesso risultano mascherate dai depositi di riempimento quaternario, e riconoscibili solamente ad una attenta analisi geomorfologica da foto aerea e di campagna. Con ogni probabilità, la differente presenza ed eterogeneità di comportamento, sia della fratturazione al suolo e del danneggiamento, può trovare ampia spiegazione appunto nella presenza di tali manifestazioni, così come ampiamente dimostrato dalle evidenze legate alla crisi sismica de L'Aquila del 2009 (Farabollini et al., 2012).

9 - variazioni di portata di alcune sorgenti. Nel corso della sequenza sismica, sono stati evidenziati importanti variazioni del regime idrico delle sorgenti presenti nell'area del cratere. Incrementi di portata importanti sono state segnalate infatti per quasi tutte le sorgenti "profonde" nell'area epicentrale (sorgente di Pescara del Tronto, di Capodacqua e di Foce di Montemonaco) alimentate dagli acquiferi dell'area dei Piani di Castelluccio e del Monte Vettore o aumenti di portata anche di molte decine di litri/secondo, come nel caso della sorgente San Chiodo di Castelsantangelo sul Nera (in corrispondenza delle ore successive al sisma) e/o temporanee alterazioni del chimismo con sporadici aumenti di torbidità dell'acqua. In alcuni casi addirittura, si è verificata la "scomparsa" della sorgente stessa come nel caso della sorgente di Forca Canapine nell'ascolano, scomparsa dopo le scosse del 26 e 30 ottobre 2016 (che contribuiva con una portata di oltre $50 \mathrm{l} / \mathrm{s}$ all'approvvigionamento idrico dei comuni del fermano e del piceno) (Aringoli et al., in stampa).

Forse l'esempio più eclatante è quello della sorgente del Torbidone (Fig. 3b), nel comune di Norcia che, scomparsa dopo il terremoto del 1979, si è riattivata dopo il 30 ottobre 2016 con una portata che è andata via via aumentando fino a raggiungere, all'inizio di febbraio, circa 2000 l/s.

\section{DATABASE COSISMICO}

Com'è nelle proprie prerogative la Regione Marche ha provveduto nel corso dell'ultimo decennio alla raccolta e alla organizzazione di dati relativi al proprio territorio, in modo da poter rispondere alle richieste provenienti da Enti pubblici e privati.

La Regione Marche è fra le poche regioni a disporre di una copertura totale di informazioni geologiche di
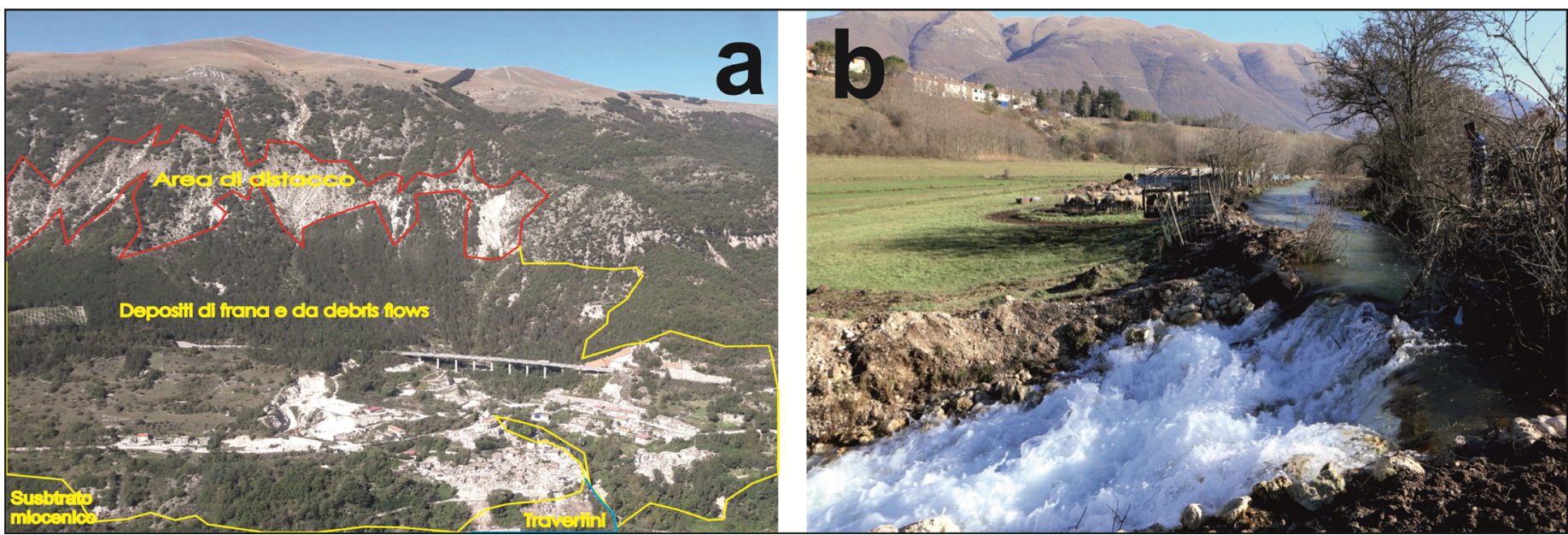

Fig.3 - a: Panoramica dell'abitato di Pescara del Tronto completamente distrutto dall'evento sismico del 24 agosto e successivamente da quello del 30 ottobre. L'ampia area di distacco sopra l'abitato è stata oggetto nel passato di imponenti fenomeni di frana e di debris flow. Alla base, poggianti su circa $30 \mathrm{~m}$ di depositi travertinosi, è stato riconosciuto, per uno spessore di circa 1-1,50m, un deposito costituito da vecchie macerie da ricondurre ai precedenti danneggiamenti subiti dall'abitato in occasione di precedenti frane e/o eventi sismcii. b: Sorgente del Torbidone (Norcia), febbraio 2017: il continuo incremento di portata ha reso necessario ricreare ed ampliare il vecchio alveo "tombato" dopo il terremoto del 1979 . 
estremo dettaglio, in buona parte derivanti dallo sforzo compiuto per contribuire alla collana della Carta Geologica d'Italia alla scala 1:50.000 - Progetto CARG e, grazie a un protocollo di intesa con Regione Emilia-Romagna, Regione Umbria e Regione Toscana, sta oggi provvedendo alla realizzazione di un continuo territoriale alla scala 1:10.000 conseguente a un complesso lavoro di omogeneizzazione. Tali dati sono pertanto in fase di integrazione entro un database relazionale apposito per la cartografia geologica a copertura dell'intero territorio delle quattro Regioni; da questo database potranno essere estratti all'occorrenza tematismi specifici e di supporto alla pianificazione, alla progettazione, all'analisi, alla ricerca.

Grazie ad alcuni progetti dedicati, gran parte della superficie della Regione Marche è inoltre coperta da dati geomorfologici altrettanto particolareggiati e anch'essi sono organizzati in una banca dati locale. In questa ottica un progetto di raccolta di nuove informazioni conseguenti alla recente crisi sismica, doveva necessariamente essere predisposto a dialogare tenendo conto delle esigenze di informatizzazione, georeferenziazione e interrogazione in relazione ai dati già esistenti (Angelini, 2014); allo stesso tempo la vastità dell'argomento Database cosismico e la molteplicità dei possibili input e segnalazioni suggerivano una interfaccia agile, con la possibilità di una continua evoluzione e aggiornamento e con opportunità di visualizzazioni e interrogazioni estremamente semplificate.

La prima fase della creazione di una banca dati consiste nell'identificazione delle entità e del dominio di conoscenza che si vuole descrivere e nel definirne le relazioni (Servizio Geologico d'Italia, 1995). I nuovi dati rilevati sono stati perciò organizzati in un database locale del tipo entità/ relazione facilmente editabile, che attraverso valutazioni complessive nel corso dei prossimi mesi possa suggerire temi di ricerca specifici, indicare criticità e suggerire aggiornamenti nella cartografia esistente (Fig. 4).

I dati sono stati inseriti come features puntuali, dotate di un identificativo (ID) progressivo e univoco all'interno del dominio territoriale grazie alle coordinate WGS'84 decimali; tale dominio è costituito dalle aree colpite dalla sequenza sismica 2016-2017. Ogni informazione registrata mantiene fra i propri attributi le caratteristiche essenziali del fenomeno e rimanda a collegamenti esterni per la tipologia della osservazione e per le informazioni accessorie. Il database utilizza concetti di entità/relazione per gestire correttamente i rapporti geografici (topologici) e informativi (attributi) relativi agli oggetti reali cartografati: è così che vengono definiti alcuni livelli informativi entro i quali vengono immagazzinate features di tipo geografico in base a "famiglie" di dati codificati; gli altri dati possono essere collegati o no all'analisi in corso a seconda dell'aspetto che si vuole evidenziare (Angelini et al., 2013).

L'applicativo propone una interfaccia semplice, operativa, entro la quale esistono spazi per la segnalazione di elementi transienti e per le note libere, oltre ovviamente a una serie di menu per la identificazione univoca dell'emergenza. La maschera di interfaccia traduce i codici utilizzati per immagazzinare le informazioni, in maniera da renderla fruibile anche senza una banca dati locale a disposizione.

Al punto di interesse inserito, sono state collegate alcune informazioni tabellari esterne specifiche, circa la litologia dei terreni coinvolti, circa lo scenario geomorfologico nel quale si inquadra la forma o il

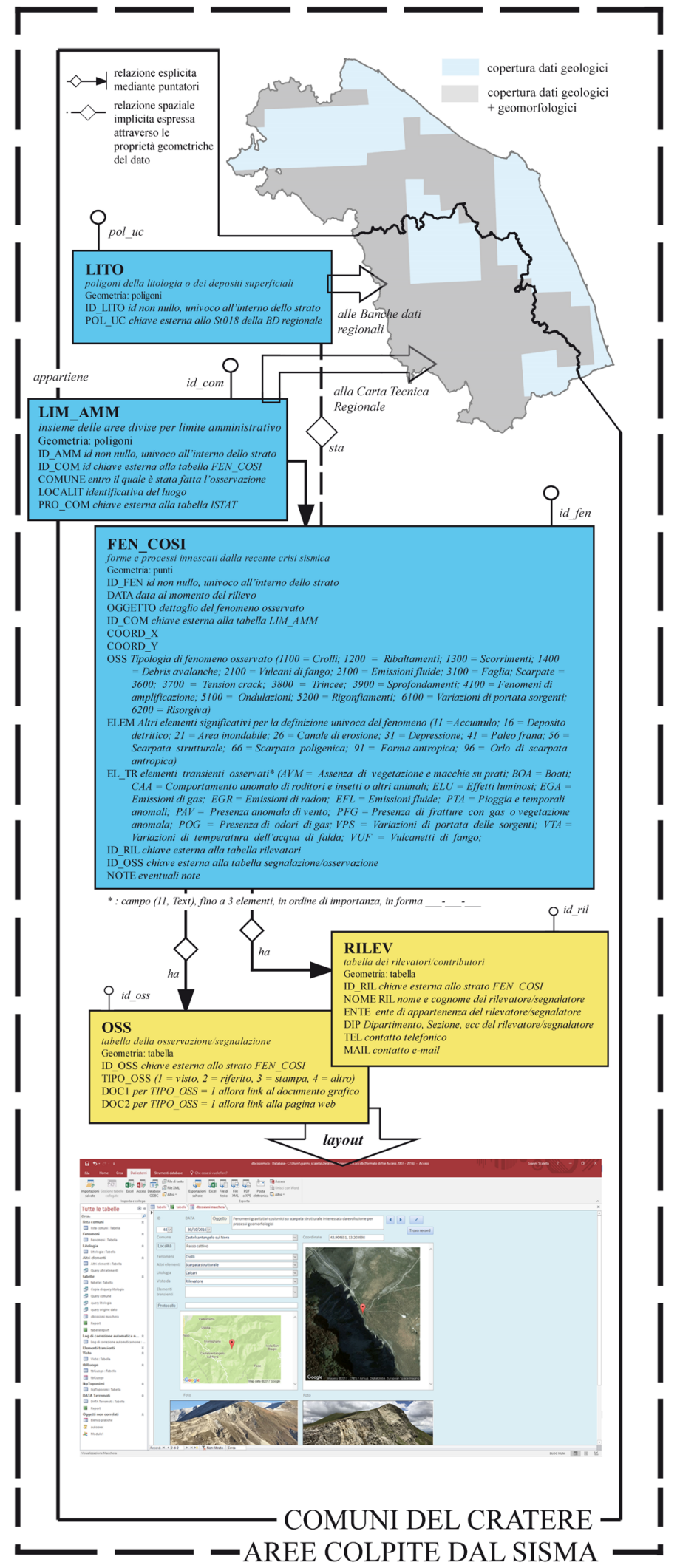

Fig. 4 - Schema logico della banca dati locale.

processo segnalato e, allo scopo di rendere immediata l'identificazione, stralci di cartografie Google, immagini puntuali e/o panoramiche. 
Le schede compilate secondo l'architettura sopra descritta, possono comunque essere utilizzate sia per la realizzazione di una nuova piattaforma integrata con i dati territoriali geologici e non, sia per il continuo aggiornamento della banca dati, sia per il monitoraggio degli aspetti morfodinamici e morfogenetici.

\section{CONSIDERAZIONI CONCLUSIVE}

L'analisi delle numerose evidenze riscontrate nell'area del cosiddetto "cratere" ha permesso di stimare che circa il 70\% degli effetti riscontrati siano ascrivibili a fratture e fessurazione cosismica; che circa il $8 \%$ siano dovute a frane s.l. ed a deformazioni, mentre il $20 \%$ circa a cedimenti di su infrastrutture viarie ed a rete ed il restante $2 \%$ a cedimenti, variazioni del regime idrico ed altre cause minori.

È tuttavia da evidenziare che la banca dati non è sicuramente completa in quanto possono essere sfuggite situazioni più o meno limitate e poco evidenti, così come pure la lunga sequenza sismica che ha dato terremoti superiori a $5 \mathrm{M}$ da agosto fino a gennaio 2017, ha spesso amplificato e sovrapposto effetti già prodotti con la prima scossa del 24 agosto, rendendo a volte difficoltoso l'acquisizione del dato.

Nella zona interessata dalla lunga sequenza sismica, il rilevamento geologico evidenzia la presenza di numerose faglie quaternarie, tutte con indizi di attività recente ed alle quali è possibile, sulla base delle loro dimensioni, attribuire un potenziale sismogenetico fino a 6.5Mw. Lungo molte di queste faglie si sono avuti fenomeni superficiali che in alcuni casi hanno ringiovanito la scarpata in roccia e/o fagliato il suolo, con freeface valutate fino ad $1 \mathrm{~m}$ (Monte Vettore).

Il problema che si pone è pertanto quello della idoneità della risoluzione dell'analisi geologica per la valutazione della pericolosità sismica di un'area: infatti, sulla base di considerazioni sui tempi di ritorno dei forti terremoti dell'appennino centrale si sarebbe potuto indicare la faglia di Norcia come la faglia sismogenetica, anziché pensare alle altre faglie che, comunque hanno espressioni morfologiche molto evidenti (faglia del Vettore, faglia di Monte Bove; faglia di Campotosto; ecc.).

Analogamente all'aspetto prettamente geostrutturale, è necessario tenere in considerazione l'effetto geomorfologico sull'ambiente e sul danneggiamento cercando di verificare e soprattutto quantificare il ruolo di alcuni elementi geomorfologici che caratterizzano il territorio dell'Appennino centrale, ed in particolare il ruolo svolto dalle grandi frane allo stato di quiescenza o alle Deformazioni Gravitative Profonde di versante, nel determinare effetti di amplificazione locale sia in bedrock che nei depositi continentali quaternari. In tal modo, sulla base degli effetti al suolo e sul danneggiamento degli edifici, sarebbe inoltre possibile, effettivamente localizzare le aree epicentrali soprattutto per quelli che vengono definiti da dati storici.

\section{REFERENCES}

Angelini S. (2014) - Banche dati e geomorfologia: esempi e applicazioni a partire da dati CARG. Atti del Convegno, Dialogo intorno al paesaggio, I, pp. 25-36

Angelini S., Farabollini P., Menotti R.M., Millesimi F., Petitta M. (2013) - Application of the compurised cartography to the territory management: the geomorphological map of palaeolandslides in the Velino River Valley. Landslide Science and Practice, Spatial Analysis and modeling, Springer, III, pp. 415-421.

Aringoli D., Bufalini M., Farabollini P., Giacopetti M., Materazzi M., Pambianchi G. \& Scalella G. (in stampa) - Effetti geomorfologici e variazioni idrogeologiche indotti dai terremoti: esempi nell'area epicentrale della sequenza sismica 2016-2017 del centro Italia. Atti Conv. Sigea, Preci, 2016.

Aringoli D., Farabollini P., Giacopetti M., Materazzi M., Paggi S., Pambianchi G., Pierantoni P.P., Pistolesi E., Pitts A. \& Tondi E. (2016) - The August 24th 2016 Accumoli earthquake: surface faulting and Deep-Seated Gravitational Slope Deformation (DSGSD) in the Monte Vettore area. Annals of Geophysics, 59(5), https://doi.org/10.4401/ag-7199.

Aringoli D., Gentili B., Materazzi M. \& Pambianchi G. (2010b) - Mass movements in Adriatic Central Italy: Activation and evolutive control factors. In: Werner, E D. and Friedman H.P., Eds., Landslides: Causes, Types and Effects, pp. 1-72.

Bourrogh P.A. \& McDonnel R.A. (1998) - Principles of Geographical Information Systems. Oxford University press.

Farabollini P., Aringoli D., Materazzi M., Pambianchi G. \& Scalella G. (2017) - Large Landslides and Deep Seated Gravitational Slope Deformation triggered by the 2016-2017 central Italy seismic crisis: first evidences from the Mount Vettore-Mt Bove epicentral area. Abs. Int. Workshop "From 1997 to 2016: Three destructive earthquakes along the central Apennine fault system, Italy". July 19-22, 2017.

Civico R., Pucci S., Villani F., Pizzimenti L., De Martini P.M., Nappi R. \& the Open EMERGEO Working Group (2018) - Surface ruptures following the 30 October $2016 \mathrm{Mw} 6.5$ Norcia earthquake, central Italy. Journal of Maps, 14(2), 151-160, https://doi.org/10.1080/174 45647.2018.1441756.

Coltorti M. \& Farabollini P. (2002) - Quaternary evolution of the Castelluccio di Norcia Basin (Umbro-Marchean Apennine, Central Italy). Il Quaternario, 8 (1), 149-166.

Farabollini P., Aringoli D., Materazzi M., Pambianchi G., Pierantoni P., Scalella G. \& Tondi E. (2012) - Il terremoto aquilano del 6 aprile 2009: rilievi geologici, geologici del Quaternario e geomorfologici e considerazioni per la prevenzione del rischio sismico e per la ricostruzione post-terremoto. Geologia Tecnica \& Ambientale, 2, 58-74.

Farabollini P., Folchi Vici d'Arcevia C., Gentili B., Luzi L., Pambianchi G. \& Viglione F. (1995) - La morfogenesi gravitativa nelle formazioni litoidi dell'Appennino centrale. Memorie della Società Geologica Italiana, 50, 123-136.

Farabollini P. \& Spurio E. (2008) - Applicazione dei GIS nello studio dei fenomeni di debris flows dell'appennino umbro-marchigiano (Regione Marche, Italia). Rendiconti Online della Società Geologica Italiana, 2, 1-9.

Pierantoni, P., G. Deiana, and S. Galdenzi (2013) - Stratigraphic and structural features of the Sibillini Mountains (Umbria-Marche Apennines, Italy). Ital. J. Geosci., 132(3), 497-520.

Servizio Geologico d'Italia (1995) - Linee guida della Carta geologica d'Italia alla scala 1:50.000, Quaderno n.6, Guida all'informatizzazione. Ed. Ist. Poligraf. e Zecca dello Stato.

Tondi E. \& Cello G. (2003) - Spatiotemporal Evolution of the Central Apennines Fault System (Italy). Journal of Geodynamics, 36, 113128. 